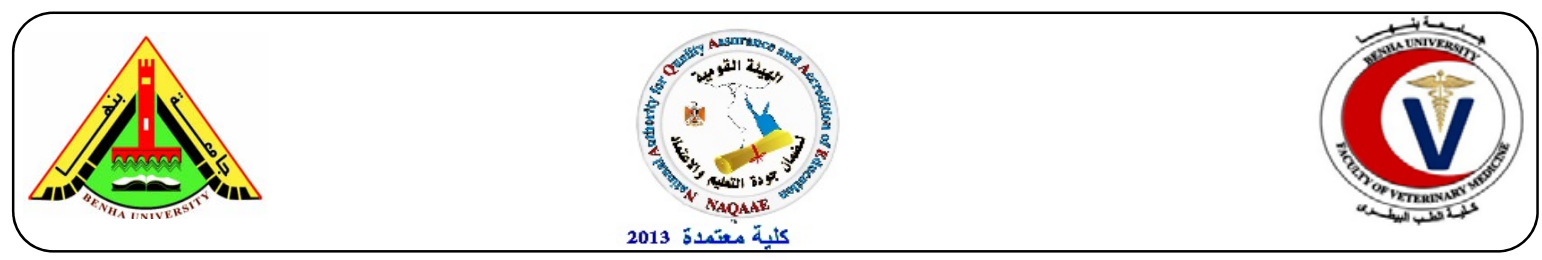

\title{
Evaluation of inactivated Rift valley fever virus vaccine adjuvanted with montanide oil ISA 201 VG.
}

\author{
Gabr F. El-Bagoury ${ }^{1}$, Saad S.A. Sharawi ${ }^{1}$, Lily S. Salama ${ }^{2}$, Marwa Y. Hammad ${ }^{2}$ \\ ${ }^{1}$ Department of Virology, Faculty of Veterinary Medicine, Benha University. ${ }^{2}$ Department of RVf, \\ veterinary serum and vaccine research institute, Abbassia, Cairo.
}

\begin{abstract}
A B S T R A C T
Inactivated tissue culture adapted Rift Valley Fever (RVF) Virus vaccines were prepared using Montanide oil ISA $201 \mathrm{VG}$ and aluminum hydroxide gel as adjuvants. The prepared vaccines were sterile and safe inducing no systemic or local clinical signs in sheep. Comparative evaluation of experimentally prepared vaccines in sheep after a single dose indicated that the oily prepared vaccines stimulated the humeral immune response as estimated by SNT and ELISA compared with aluminum hydroxide gel vaccine. Protective serum antibody titers against in Montanide oil ISA 201 VG adjuvenated RVF vaccine appeared in protective level at 1st week post vaccination and aluminum hydroxide gel adjuvenated RVF vaccine at 2 nd week. This protective titer persisted till 12 th month in the new oily vaccine and till the 6th month for Aluminum hydroxide gel vaccine. Both of SNT and ELISA indicated that among the used adjuvant Montanide oil ISA 201 VG was preferable.
\end{abstract}

Keywords: RVF virus vaccines, Adjuvants, SNT, ELISA.

(http://www.bvmj.bu.edu.eg)

(BVMJ-29(1): 220-223, 2015)

\section{INTRODUCTION}

$\mathrm{R}$ ift Valley fever (RVF) virus, a Phlebovirus from the family Bunyaviridae, is potentially transmitted by many different species of insect vectors that have a wide global distribution (Gubler, 2002). Periodic RVF outbreaks in livestock (goats, sheep, cattle, and camels) and acute febrile illness with hemorrhagic syndrome in humans have been reported widely throughout south and central Africa, from Kenya westward into Nigeria, Niger, Burkina Faso, Senegal, and Mauritania and northward into Egypt (Diallo et al., 2005). To limit spread of the disease, veterinary vaccines are the first line of defense against RVF virus infection. Extensive work has been carried out to produce safe and efficient vaccines against Rift Valley Fever (Kamal, 2011). A trial for preparing a potent and safe inactivated vaccine to be used for the spreading of the disease was attempted (Abd El Samea et al.,
1994, El-Bagoury et al., 2013). Two types of inactivated RVF vaccines were produced in Egypt; first produced by VACSERA company which is formalin inactivated and alum adjuvanted (Menya/Sheep/258), and the second produced by the Veterinary Serum and Vaccine Research Institute (VSVRI) which is binary ethylenimine (BEI) inactivated and alum hydroxide adjuvanted (ZH501strain) (El-Nimr 1980) and (Eman 1995). The present trend in the development of new vaccines focuses on simplification of vaccination schemes either by increasing the components in the vaccines or by decreasing the number of vaccination doses needed to impart sufficient protection. One of the possibilities how to achieve this goal is the use of new, more effective adjuvants. Adjuvant substances have been used for almost 80 years for increasing the effectiveness of various vaccines (Ramon, 1925). For a long time, oil adjuvants based on incomplete Freund's adjuvant played an 
important role in commercial veterinary vaccines. However, the mineral oil used caused various post-vaccination reaction of local or even general character in vaccinated individuals (Vanselow, 1987). After replacing mineral oils with metabolisable natural oils (soya, sesame, olive, etc.) some local reactions were eliminated (Reynolds et al., 1980, ElBagoury et al., 2013). At the present time aluminium hydroxide is the substance most frequently used as adjuvant in veterinary medicine (Clements and Griffiths, 2002) although its potentiating effect fails to reach in general the level of oil adjuvants. The present investigation dealed with the use of Montanide ISA $201 \mathrm{VG}$ oil $\mathrm{W} / \mathrm{O} / \mathrm{W}$ as adjuvant with RVF virus inactivated vaccine in order to induce high prolonged potent immunity in vaccinated sheep.

\section{MATERIAL AND METHODS}

\subsection{Virus strain}

RVF virus Zagazig Human 501 (ZH501) strain was propagated in BHK-21 cell line of a titer 107.5 TCID50/ $\mathrm{ml}$ was supplied by RVF Department, Veterinary Serum and Vaccine Research Institute, Abbasia, Cairo. It was used for preparation of inactivated vaccine, SNT and ELISA.

\subsection{Cell lines}

Baby hamster kidney (BHK-21) cell line was established by Mackpherson and Stocker, 1962. They were used for propagation, titration of the virus and testing the safety of prepared inactivated virus suspension.

\subsection{Animals}

\subsubsection{Mice}

Forty five Albino Swiss mice, 3- 4 weeks old, were supplied by the Department of Pet Animal vaccine Research; Veterinary Serum and Vaccine Research Institute; Abassia, Cairo. These mice were used in toxicity test to determine safe concentration of oil adjuvants.

\subsubsection{Sheep and experimental design}

Twenty four local breed sheep 3-4 month old were used for evaluation of their immune response to the prepared vaccines. All of these animals were screened using SNT and proved to be free from RVF antibodies, the sheep were divided into 4 groups as follows:-

Group 1(G1): six animals, each vaccinated subcutaneously (S/C) with $1 \mathrm{ml}$ of inactivated Montanide oil ISA 201 RVF vaccine.

Group 2(G2): six animals, each vaccinated subcutaneously $(\mathrm{S} / \mathrm{C})$ with $1 \mathrm{ml}$ of inactivated aluminium hydroxide gel RVF vaccine.

Group 3(G3): six animals kept as non vaccinated (control negative).

\subsection{Samples collection}

All sera were collected from groups 1, 2, 3, 4 on the day of vaccination (zero day), then weekly till 4 th week post vaccination and monthly till protective antibody level declined. The sera were stored at $-20^{\circ} \mathrm{C}$ and inactivated at $56^{\circ} \mathrm{C}$ for 30 minutes before being examined by the Serum Neutralization Test (SNT) and indirect enzyme-linked immunosorbent assay (ELISA).

\subsection{Adjuvants}

Montanide oil ISA201 VG was obtained from Seppic, Paris, France. Aluminium hydroxide gel was obtained from (Alliance Bio Company, USA), Lot. No. 11-274-30 obtained from (Alliance Bio Company, USA), Lot. No. 11-274-30.

\subsection{Serum neutralization test (SNT)}

It was performed using the micro technique as described formerly (Ramon G. 1925).

\subsection{Enzyme-linked immunosorbent assay (ELISA)}

It was carried out according to earlier author (Randall et.al. 1964) to determine antibodies against RVF virus using ELISA. 


\section{RESULTS}

\subsection{Sterility test}

Inactivated vaccines were sterile as they were free from any bacterial and fungal contaminants.

\subsection{Safety test of inactivated RVFV in baby mice}

Inactivated vaccines were safe. no mortality occurred indicates optimum inactivation process.

\subsection{Humoral immune response in sheep vaccinated with inactivated RVF virus vaccines}

Serum neutralizing antibody titer against RVF virus were detectable at the protective level by 1 nd week post vaccination in GP 1 , while the protective titer induced by GP 2 was detectable by 2 nd week post vaccination. It showed that the mean neutralizing index in Gp.1 reached above the protective level at the $1^{\text {nd }}$ week post vaccination and increased gradually till reached the peak at the $4^{\text {rd }}$ month post vaccination and still within the protective level till the end of experiment.

It also showed that the mean neutralizing indeces in sheep vaccinated with inactivated RVF vaccine (Aluminum hydroxide gel) Gp.2 reached to the protective level at the $2^{\text {nd }}$ week post vaccination and increased gradually till reached the peak at the $2^{\text {nd }}$ month post vaccination then the level decreased at the $6^{\text {th }}$ month post vaccination and decline to a non protective level.

RVF virus antibodies titer expressed with the optical density in sera of vaccinated sheep as observed by ELISA found parallel to preceding result in SNT.

Fig .1. Mean neutralizing indices at different period post vaccination.

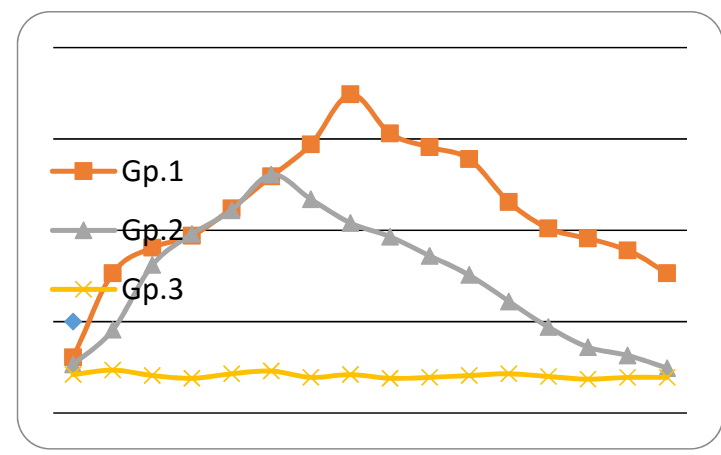

Group (1) sheep vaccinated by inactivated RVF vaccine with Mtn. ISA201, Group (2) sheep vaccinated by inactivated RVF vaccine with with Alum. Hydroxide, Group (3) Control non vaccinated sheep

Fig .2. Mean values of ELISA optical density indices at different period post vaccination.

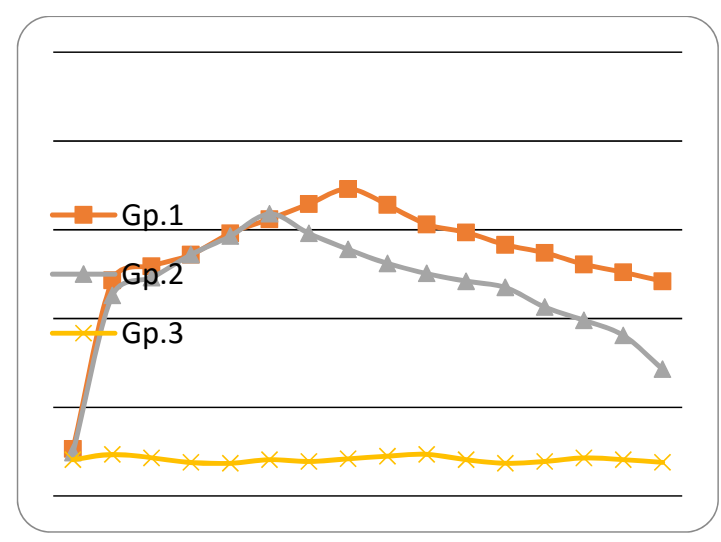

Group (1) sheep vaccinated by inactivated RVF vaccine with Mtn. ISA201, Group (2) sheep vaccinated by inactivated RVF vaccine with Alum. Hydroxide, Group (3) Control non vaccinated sheep.

\section{DISCUSSION}

Often vaccinologists search and aim to improve vaccines to overcome the obstacles which may face the older vaccines such as the unsafely or the low induced immunity. The use of adjuvant plays the greater role in this field. The present work is a trial to improve the locally produced inactivated RVFV vaccine through the use of two different adjuvants other than the used aluminum hydroxide gel.

The experimental results revealed that all prepared vaccine formula were free from foreign contaminants (aerobic and anaerobic bacteria; fungi and mycoplasma) and safe in vaccinated animals where such 
animals remained healthy all over the experimental period without local reaction at the site of inoculation. These observations agree with the recommendations of (USA-CER 1987).

Results of SNT and ELISA were coming in a parallel manner confirming each other. In conclusion, results gave the priority to the prepared inactivated Montanide oil ISA61 RVF vaccine and inactivated Montanide oil ISA201 RVF vaccine over the local inactivated aluminum hydroxide gel adjuvanted RVF vaccine beside that RVF vaccine with Montanide oil ISA201 induces rapid onset immunological response with long duration (El-Bagoury et al., 2013). These adjuvants are patent contain its own surfactant which enable an easy manufacturing of vaccines by mixing the aqueous medium into the montanide oil at room temperature.

\section{REFERENCES}

Abd El Samea, M. M.; Elian, K. and Gihan, K. M. 1994. The effect of Rift Valley Fever and sheep pox vaccines on the immune response of sheep. J. Egypt. Vet. Med. Ass. 2:129-136.

Clements CJ, Griffiths E. 2002. The global impact of vaccines containing aluminum adjuvants. Vaccine; 20:24-33.

Diallo, M., Nabeth, P., Ba, K., Sall, A., Ba, Y., Mondo,M., Girault, L., Abdalahi,M. and Mathiot, C. 2005. Mosquito vectors of the 1998-1999 outbreak of Rift Valley Fever and other arboviruses (Bagaza, anar, Wesselsbron and West Nile) in Mauritania and Senegal. Med. Vet. Entomol. 19:119-126.

El-Bagoury, G.F., El-Nahas, E.M., Haassan, K. Z, Youssef, M. M. 2013. Comparative evaluation of prepared inactivated Rift valley fever virus vaccine with different adjuvants. BVMJ 24(2):106-114.

El-Nimr, M.M. 1980. Studies on the inactivated vaccine against RVF. Ph. D. Thesis (Microbiology). Fac. Vet. Med Egypt: Assiut University.

Eman. M. S. S. 1995. Studies on RVF vaccine inactivated with Binary. Ph. D. Thesis (Microbiology). Fac. Vet. Med Egypt: Cairo University.

Gubler, D. J. 2002. The global emergence/resurgence of arboviral diseases as public health problems. Arch. Med. Res. 33:330-342.

Kamal, S.K. 2011. Observations on rift valley fever virus and vaccines in Egypt. Virol. J. 8:532-540.

Ramon G. 1925: Sur l'augmentation anormale de l'antitoxine chez les chevaux producteurs de serum antidiphtérique. Bull Soc Centr Méd Vét. 101:227-234.

Mackpherson, L.A. and Stocker, M.G.P. 1962. Polyoma transformation of hamster cell colonies; An investigation of genetic factors affecting cell competence. Virology, 16: 147.

Randall, R.; Binn, L. N. and Harison, V. R. 1964. Immunization against Rift Valley Fever virus. Studies on the immunogenicity of lyophilized formalin inactivated vaccine. J. Imm., 93(2):293299.

Reynolds JA, Harrington DG, Crabbs CL, Peters CJ, Di Luzio NM. 1980. Adjuvant activity of a novel metabolisable lipid emulsion with inactivated viral vaccines. Infect Immun; 28:937-943.

USA-CER 1987. Code of Federal Regulation; United States of America.

Vanselow BA. 1987. The application of adjuvants to veterinary medicine. Vet Bull. 57:881-96. 